\title{
Inherent Flaws in Login Systems of Facebook and Twitter with Mobile Numbers
}

\author{
Jawaid A. Mangnejo ${ }^{1,}{ }^{*}$, Arif R. Khuhawar ${ }^{2}$, Muneer A. Kartio ${ }^{1}$ and Saima S. Soomro ${ }^{3}$ \\ 'Department of Computer Science, Shah Abdul Latif University Khairpur, Pakistan \\ mangnejo.jawaid@gmail.com; kartiomuneer@gmail.com \\ ${ }^{2}$ School of Computer Science and Technology, Beijing Institute of Technology, China \\ arifrazakh@gmail.com \\ ${ }^{3}$ International Association for Educators and Researchers, UK \\ saima.s.soomro@gmail.com \\ *Correspondence: mangnejo.jawaid@gmail.com
}

Received: $15^{\text {th }}$ July 2018; Accepted: $6^{\text {th }}$ August 2018; Published: $1^{\text {st }}$ October 2018

\begin{abstract}
There are many social networking websites that provide online networking opportunities; all of these platforms require creating an account, then logging in and updating information to maintain a profile. Our research investigates mobile number based log in feature of both Facebook and Twitters - two exemplary social networking sites - for degree of seamlessness and other security features. Our research reveals that there are major dissimilarities as well as flaws in the deployment of mobile number based login on these two platforms. It has been observed that Twitter allows creation of multiple accounts using one single mobile number, however, logging out from one such account automatically logs out all other accounts associated with the same number. On the contrary, Facebook allows creation of multiple accounts using one single mobile number without deactivation of the previously created ones, however, if same password is used for more than one accounts associated with the same number, only one account can be accessed at any given time. This paper also provides probable solutions to address the security loopholes as identified by our research.
\end{abstract}

Keywords: Social Networking Platforms; Facebook; Twitter; Login System; Mobile Number; Password; Security

\section{Introduction}

Internet has been playing a vital role in many aspects of our lives including personal and societal ones. People from all around the globe are now connected through the Internet; according to the Internet Live Stats the number of connected people is estimated to be 4,030,740,950 in 2018 with an approximately $7.5 \%$ increase every year [1]. To communicate with friends and family using the Internet, social networking platforms such as Facebook, Twitter, Whatsapp are amongst the most popular ones. There are approximately 2.23 billion active users on Facebook as shown in Fig. 1, which are increased by $17 \%$ per year [2]. Amongst the active Facebook users, there are $47 \%$ male and $53 \%$ female as shown in Fig. 2. On the contrary, there are approximately 335 million active users on Twitter 
as shown in Fig. 1, which are increased by $14 \%$ per year [3]. Amongst the active Twitter users $42 \%$ are male while $58 \%$ are female, as shown in Fig. 2.

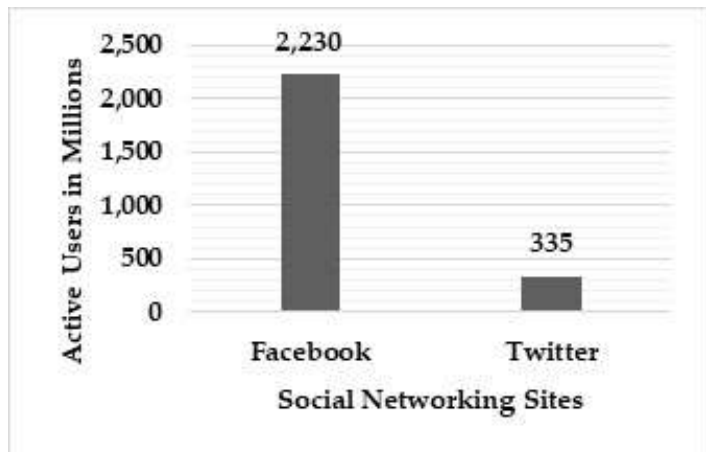

Figure 1. Statistics of Active Users in Social Networks.

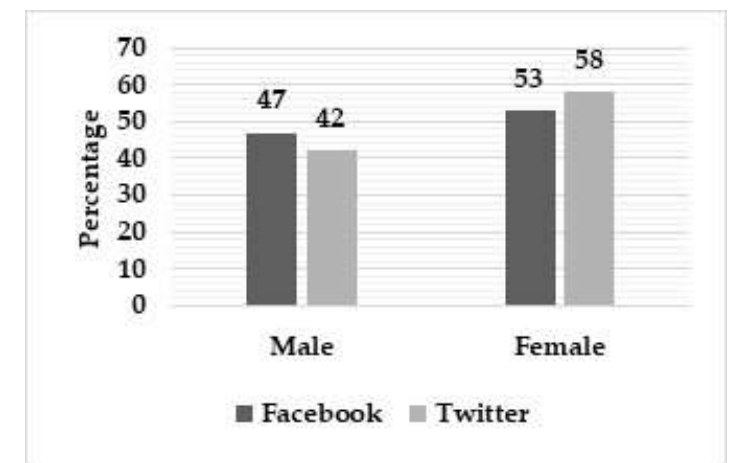

Figure 2. Statistics of Gender Breakdown in Social Networks.

To take advantage the facilities offered by any social networking platform, a user must create an account according to constraints provided by social networking sites, such as email address as a user name and password containing at least six digits for the purpose of security and authenticated login [4]. Several platforms, such as Instagram, Twitter, VK, and Facebook, provides higher flexibility by letting mobile phone numbers to be used as usernames. Since the social networking sites hold and have access to many sensitive information of the users, they are professionally, ethically as well as legally responsible to provide secure environments for the users. However, many security breaches have been recorded so far.

Since the launching of Facebook and Twitter, these platforms collect a lot of personal information from the users, which put them in risk. Both the platforms faced many serious security issues. Amongst these, accounts duplication or cloning, accounts hijacking, malware, phishing and social engineering attacks seem to be common by Elinor Mills [5]. Both social networking sites also contain hazardous bugs. Some of these bugs are directly concerned with the login systems of Facebook and Twitter because account overlapping may happen during the login process. Most of the social networking sites requires unique email address or mobile number for the purpose of security and authentic login. However, Facebook and Twitter do not require such uniqueness while using mobile number as username. Due to lack of this uniqueness, users can create multiple accounts with same mobile number. Facebook users can create multiple accounts using the same mobile number as long as the passwords for each such accounts are different. It has been found that if the password of one account is updated which matches with the password of another account then access and control to the later will be lost. It is observed that Facebook rather focuses and checks the uniqueness of password for secure and authentic login instead of mobile number while creation of multiple accounts using the same mobile number. Moreover, Facebook does not allow users to update the mobile number using the account settings for the accounts created using mobile numbers. Furthermore, it is also observed that users mostly provide the same password because it is quite easy to remember in the future.

The Twitter users are also allowed to create multiple accounts with same mobile number. If one account is created with mobile number and the user wish to create another account with the same mobile number, the second account is also created successfully without any security checks. However, when user logged out from one account then user becomes unable to access both of the accounts. In such cases, users even cannot login or recover any of the accounts as it simply redirects to the error page. As part of this research project, other social networking sites such as VK and Instagram were also tested with similar procedure but no bug was found.

\section{Social Networking Websites}


Social networking services are an online platform where people make new relations of interest, contact with family and friends and share information amongst one another. More than 200 social networking websites are available now amongst which Facebook, Twitter, LinkedIn, Google+, YouTub are commonly popular amongst all age of groups of users. Facebook, Twitter and YouTube are most widely used social networking websites amongst them.

\subsection{Facebook}

Facebook is most attractive and popular social networking site which have most active users than other social networking sites [6]. It provides attractive and user friendly interface to share images, videos and other important information among friends and family. It also provides with a platform to send messages to loved ones with the help of particular account. There is no monetary cost of having a Facebook- it is free. However, similar to other platforms, Facebook mainly generates the business by advertising and utilising users' data - considered to be the fuel of next generation a data driven world.

\subsection{Twitter}

Twitter let users know what is happening in the world right now. It is a microblogging social networking site where user follow the tweets of their own interests. Twitter allow user to share message of 280 characters or less in one tweet. This tweet is shared among the Twitter community followers or followed. Registration of accounts on Twitter is free like Facebook, users have to provide their email address or mobile number for successfully creation of accounts.

\subsection{Facebook and Twitter Login System}

The login system is a basic method of authentication to access user accounts securely. Facebook and Twitter login systems authenticate users through three methods using email IDs, mobile number, and user ID with password constraints.

1. Login with Email Address: Using email address is a hassle free approach to login into any system, such as Facebook or Twitter platforms, as it preserves uniqueness since is not possible to create more than one account using same email address. Thus email address serves as a unique identifier in any account.

2. Login with Mobile Number: Mobile numbers can also be used as a mean of account creation instead of email addresses. However, this may not be a safer choice for Facebook or Twitter platform as both these social networking sites are not strict in maintaining username uniqueness for login with mobile number. However, other social networking sites such as Vk and Instagram are enforcing uniqueness in mobile number login system.

\section{Research Methods}

The principle aim of this research is to analyse Facebook and Twitter websites account creation and authentication systems, especially from security aspects. For this purpose, both Facebook and Twitter were investigated by creating and login into multiple accounts by using both email address and mobile number seperately. Results of each scenario was then collected and analysed, as disclosed in the results section (see section 4 ).

\subsection{Data Collection}

Data were gathered while creating and login into Facebook as well as Twitter accounts with mobile numbers and email addresses; different snapshots were taken during these processes and then careful analysis was made on Facebook and Twitter login systems. The total number of dummy accounts created on Facebook are twelve, using four mobile numbers such as 03152867089, 03363724089,03340033363 and 03313410245 by creating three accounts with each mobile number but 
with differences in profile names and passwords. Mobile number 03152867089 was used to create accounts "Hamza Ali", "Zarar Hussain" and "Akeel Ahmed" pairing with passwords "Hardware@1", "Hardware@2" and "Hardware@3" respectively. Mobile number 03363724089 was used to create accounts "Sajad Ali", "Abdul Rehman" and "Maria Parveen" pairing with passwords "Hardware@1", "Hardware@2" and "Hardware@3" respectively. Mobile number 03340033363 was used to create accounts "Simran Gupta", "John Mathew" and "Sonia Arif" coupling with passwords "Hardware@1", "Hardware@2" and "Hardware@3" respectively and mobile number 03313410245 was used to create accounts "Asim Hussain", "Arman Ali" and "Saima Ahmed" coupling with password “Hardware@1", “Hardware@2” and “Hardware@3” respectively.

Same procedure was applied on Twitter too; the total number of accounts created on Twitter are ten, using five mobile numbers such as 03152867089, 03363724089, 03461323253, 03340033363 and 03313410245 by creating two dummy accounts with each mobile number but with differences in profile names and passwords. Mobile number 03152867089 is used to create accounts "Hamza Ali" and "Zarar Hussain" pairing with passwords "Software@1" and "Software@2" respectively. Mobile number 03363724089 was used to create accounts "Sajad Ali" and "Abdul Rehman" coupling with passwords "Software@1" and "Software@2" respectively. Mobile number 03461323253 was used to create accounts "Feroz Khan" and "Humera Parveen" pairing with passwords "Software@1" and "Software@2" respectively. Mobile number 03340033363 was used to create accounts "Aisha Ahmed" and "John Mathew" coupling with passwords "Software@1" and "Software@2" respectively. Mobile number 03313410245 was used to create accounts "Saima Ahmed" and "Asim Hussain" pairing with password Software@1 and Software@2 respectively. Our analysis of the results found several flaws in Facebook as well as Twitter login systems.

\section{Results}

This section presents the results of flaws found in Facebook and Twitter login systems using same mobile number. The following process depicted in Figs. 3-5 below, is applied on all the accounts that are shown in Table 1 and Table 2 during creation, login and authentication and update processes. Initially, several Facebook accounts were created using similar mobile numbers but differences in accounts are maintained in profile names and password constraints provided during the creation of accounts.



Figure 3. Creation of Simran Gupta account with same mobile number.

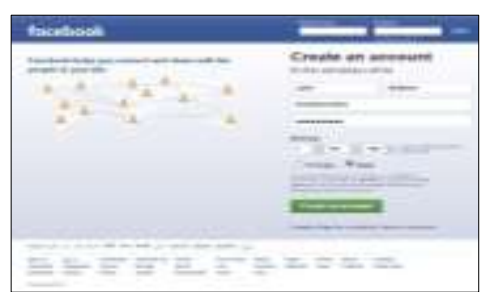

Figure 4. Creation of John Mathew account with same mobile number.

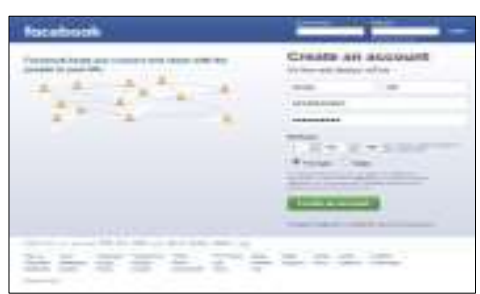

Figure 5. Creation of Sonia Arif account with same mobile number.

Once the required fields were filled-in, then the next step was the creation of accounts. Facebook sends a security code to the mobile number for verification and waits for insertion of the verification code from the user into the given field as shown in Figs. 6-8. However, this social networking platform does not check whether an account has already been created with the same mobile number or not. Thus, all accounts are successfully verified after the confirmation message as shown in Figs. 9-11. 


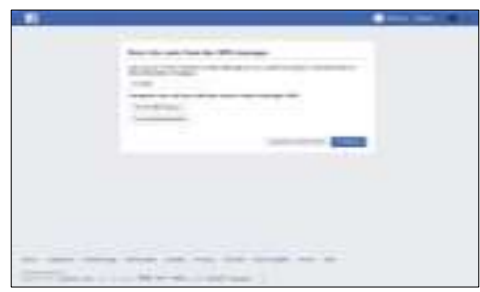

Figure 6. Code verification of Simran's account through same mobile number.



Figure 9. Simran's account code successfully verified with same mobile number.

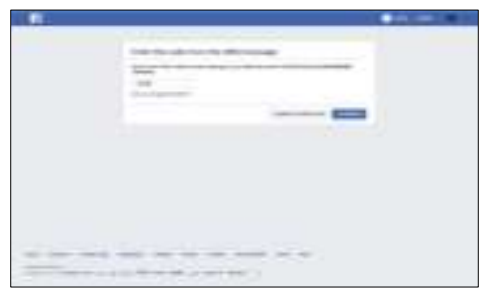

Figure 7. Code verification of John's account through same mobile number.

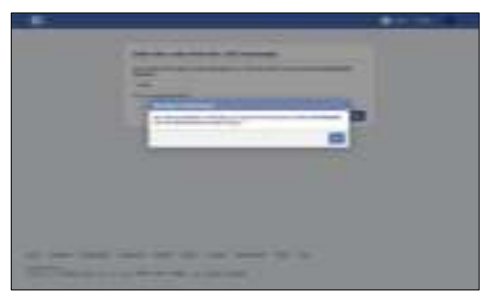

Figure 10. John's account code successfully verified with same mobile number.

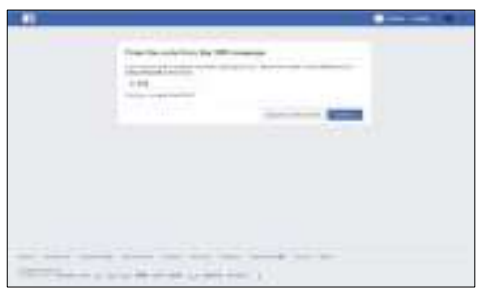

Figure 8. Code verification of Sonia's account through same mobile number.

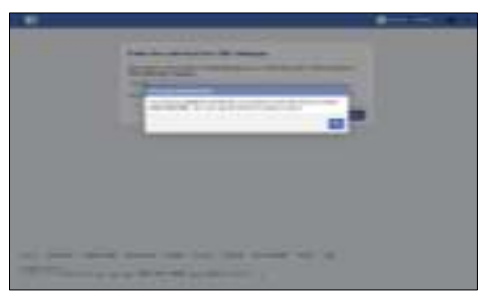

Figure 11. Sonia's account code successfully verified with same mobile number.

After confirmation and verification of the code, the successfully created Facebook accounts with the same mobile number (as shown in Figs 12-14) without any hindrance, Facebook also allow the different users to login with same mobile number simultaneously.

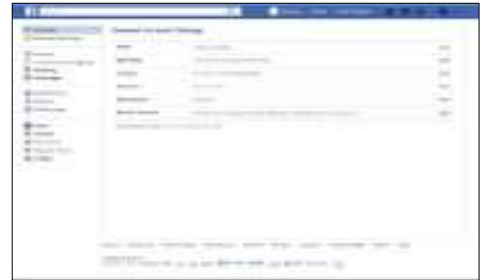

Figure 12. Simran's account is successfully created.

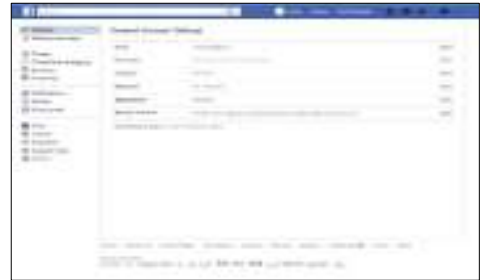

Figure 13. John's account is successfully.

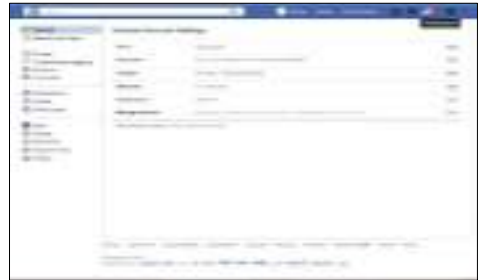

Figure 14. Sonia's account is successfully created.

It is observed that Facebook differentiate accounts by password constraints. If the user changes or inserts new password to any of the accounts created with same mobile number and if that password is matched with already assigned password of any account associated with the same mobile number then Facebook accounts are overlapped and only one account can be accessed by the user.

The Twitter also allows users to create multiple accounts with the same mobile number simply by filling the required fields as shown in Figs 15-16 and sign up for an account as shown in Figs. 1718.

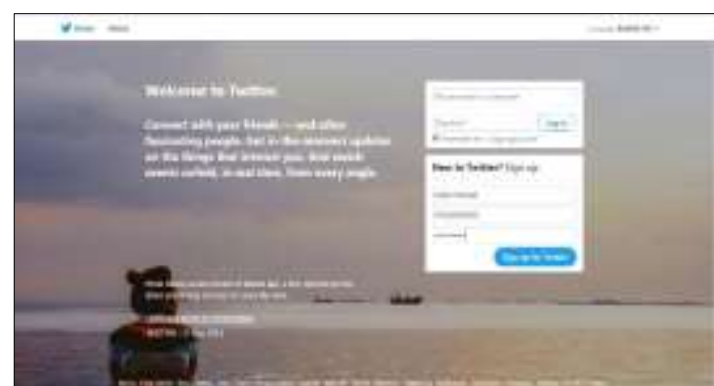

Figure 15. Creation of Aisha Ahmed account with same phone number.

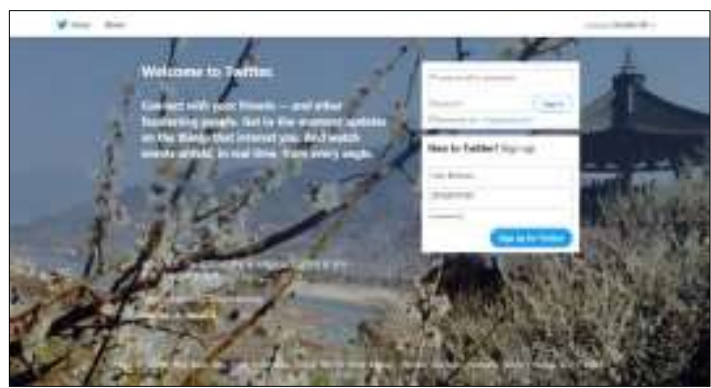

Figure 16. Creation of John Mathew account with same phone number. 


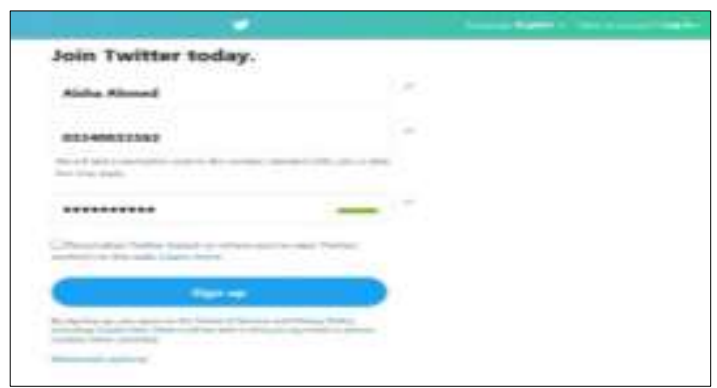

Figure 17. Sign up for Aisha Ahmed's Account with same phone number.

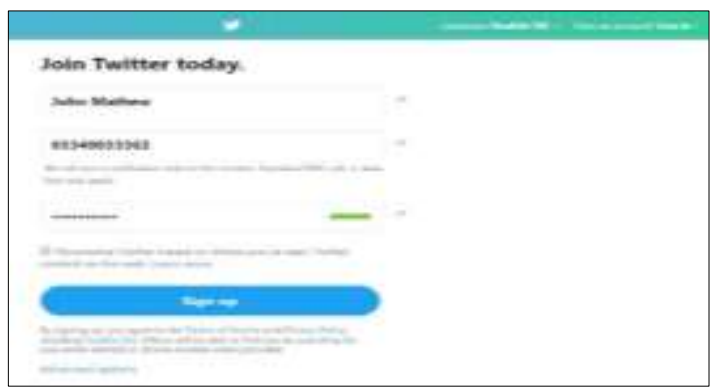

Figure 18. Sign up for John Mathew's account with same phone number.

As like Facebook, Twitter also send verification code by SMS for security checks. However, they also do not perform any security checks whether the same mobile number has been already been used or not, as shown in Figs. 19-20.

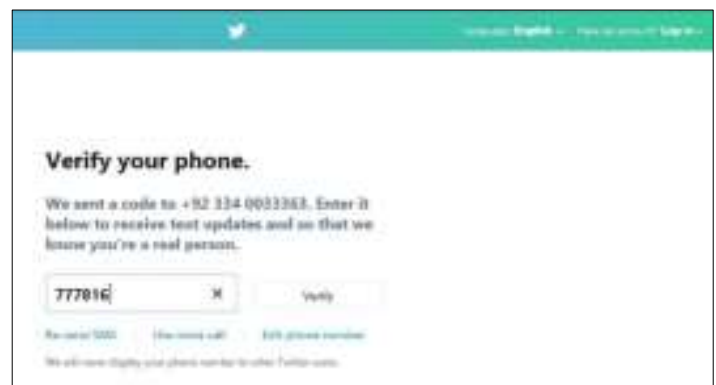

Figure 19. Code verification of Aisha's account with same phone number.

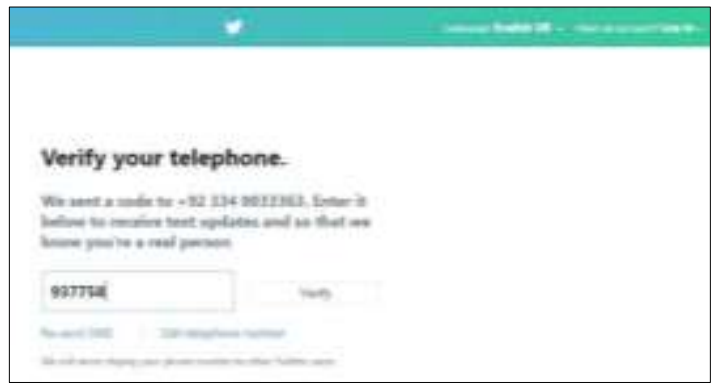

Figure 20. Code verification of John's account with same phone number.

After code confirmation, the accounts are successfully created on Twitter with the same phone number without any restriction as depicted in Figs 21-22. However, if the user is logged out from an account, then the user cannot further login in any of the accounts. The system displays an error message that multiple accounts are in existence with similar phone number as shown in Figs. 23-24.

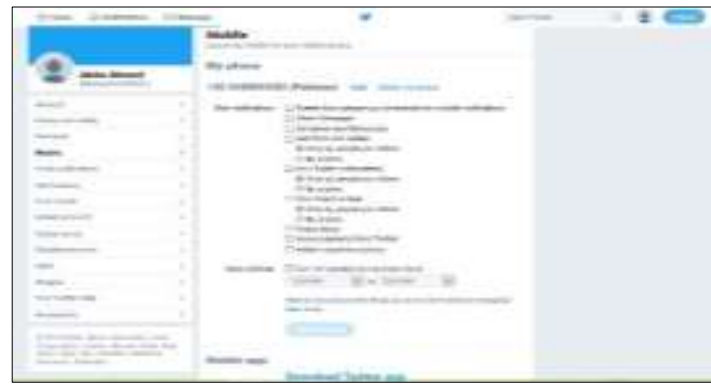

Figure 21. Aisha Ahmed's account is successfully created with same phone number.

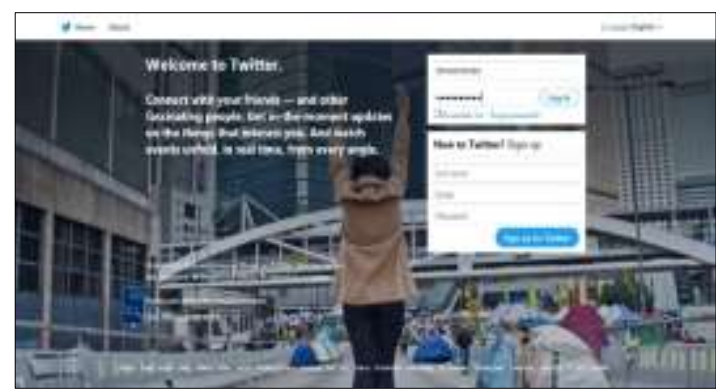

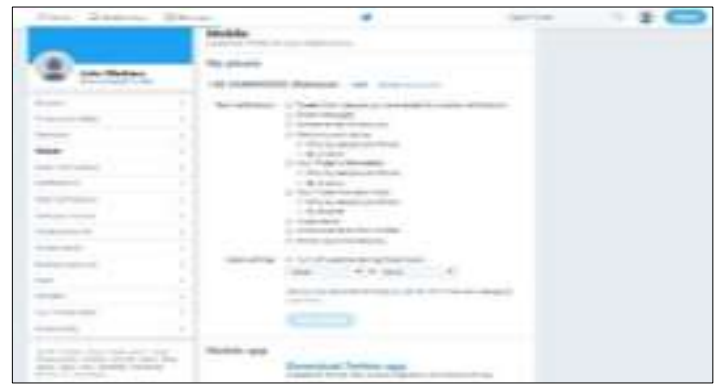

Figure 22. John Mathew's account is successfully created with same phone number.

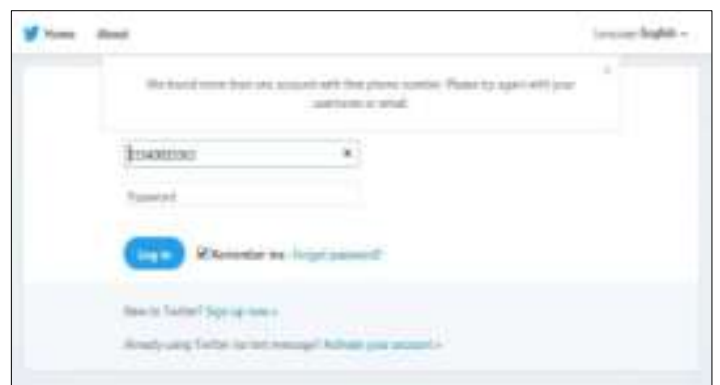


Figure 23. Login into Twitter account with phone number.
Figure 24. Error message during login into Twitter account with phone number.

When the users fail to login into Twitter account, Twitter provides the users with an option to activate the account simply by clicking on "Activate your account" as shown in Fig. 24. After clicking on the given option, the system redirects to another page as shown in Fig. 25. Now it is required to insert the phone number into the given field and click the submit button to activate the account but Twitter could not find any account associated with the provided phone number. It rather displayed an error message as shown in Fig. 26.

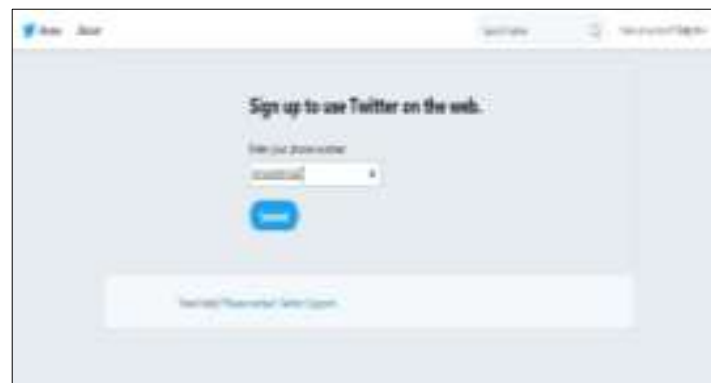

Figure 25. Try to activate Twitter account with phone number.

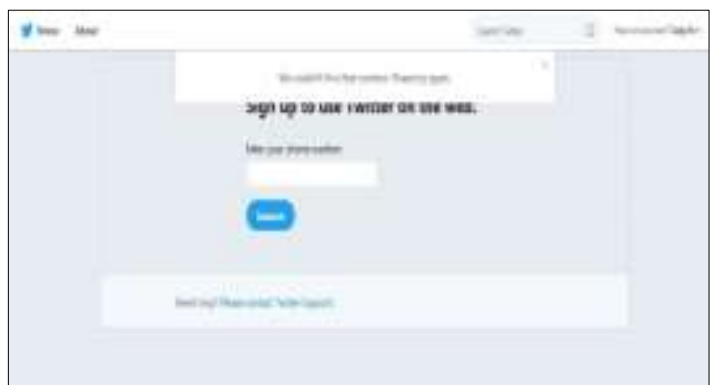

Figure 26. Error message during activation of account with phone number.

When the user tries to recover the account by selecting the forgotten password option and then supplying the phone number, Twitter cannot match any account associated with that mobile number. It rather shows the error message, as shown in Figs. 27-28, even though multiple accounts were already created using the provided mobile phone number.

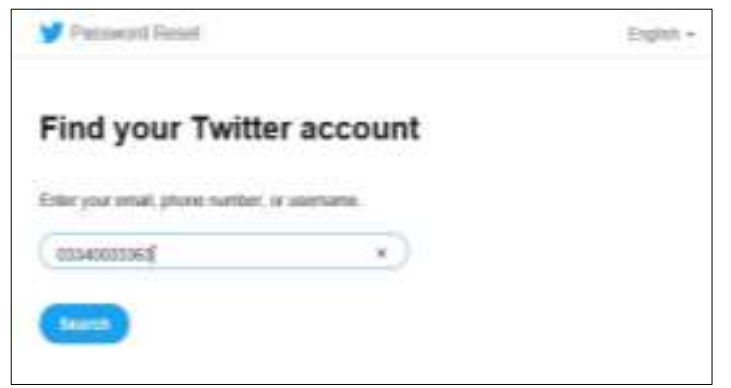

Figure 27. Recovery of account from forgot password with phone number.

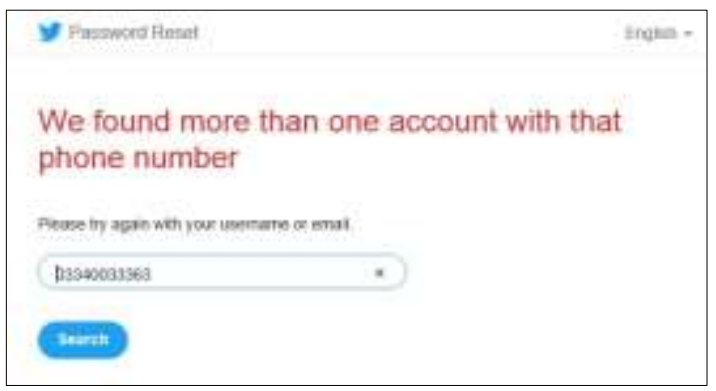

Figure 28. Error message during recovery of account with phone number.

\section{Discussion}

On Facebook platform, multiple accounts were created using the same mobile number but with differences in passwords. Whenever the user changes the password of an account matching with the password of another account associated with the same mobile number then the accounts are overlapped and only one account can be accessed. In this case, if user uses the forgot account option supplying the mobile number for finding an account as shown in Fig. 29, multiple accounts appear that were created with the provided mobile number. From the result that appeared on the screen, when the user choose any account by selecting "this is my account" option, as depicted in Fig. 30, it redirects to "I cannot access my email account" option as shown in Fig. 31. However, since the user did not provide any email address during or after the creation of the accounts, so the user cannot recover it because the accounts are created only with mobile number, it redirects to "No email access" page as shown in Fig. 32. 


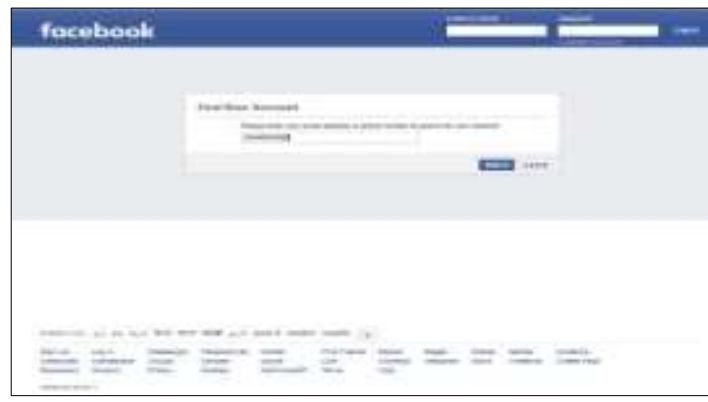

Figure 29. Find an account from forgetten password with mobile number.

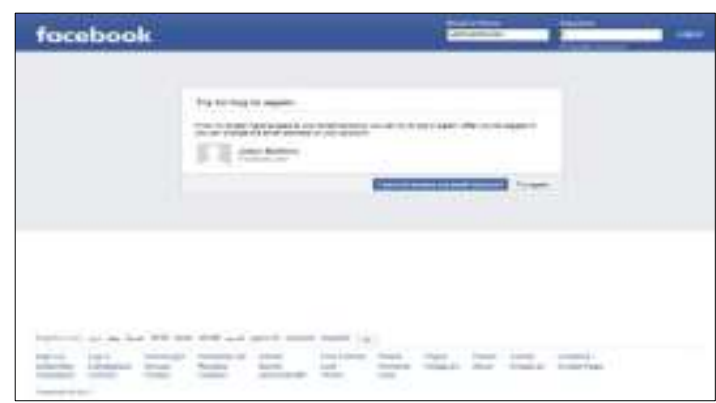

Figure 31. I cannot access my email account even no email provided.

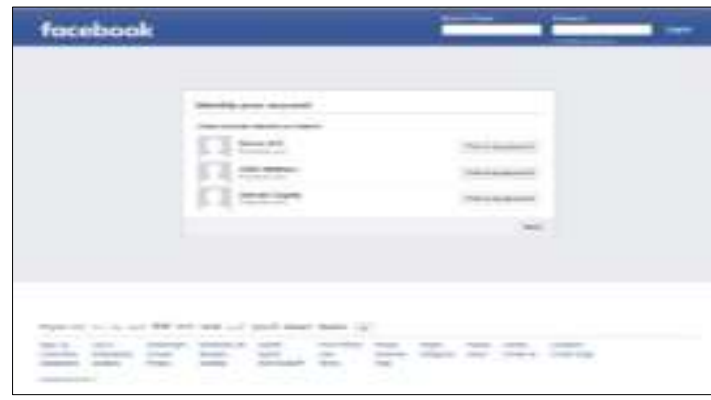

Figure 30. Multiple accounts are displayed with single mobile number.

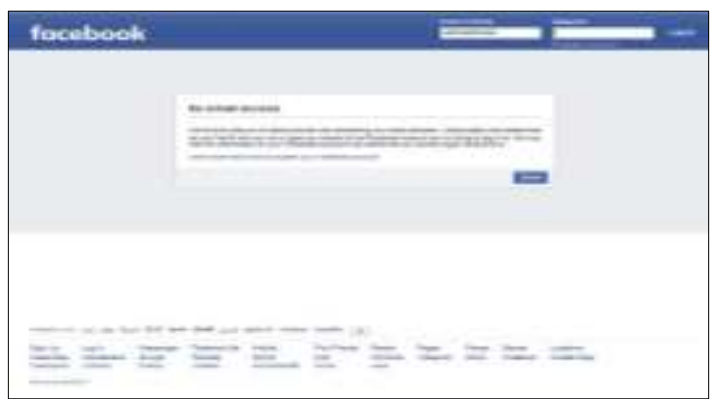

Figure 32. No email access even no email provided.

Furthermore, if the Facebook user tries to change the mobile number by visiting settings option and if that mobile number is also used by another account, Facebook alerts the user by message stating that the number is already in use and demands for another mobile number, as shown in Fig. 33. The account deactivation option is missing from the account settings, as shown in Fig. 34, thus the user is unable to deactivate the account. This may lead further to complicacy.

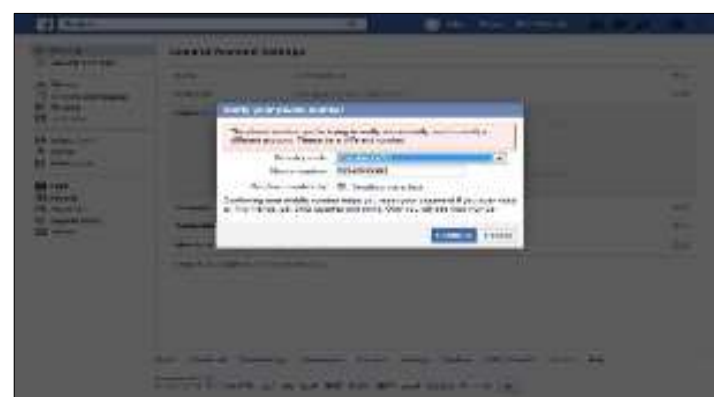

Figure 33. Enforcing mobile number uniqueness in settings only.

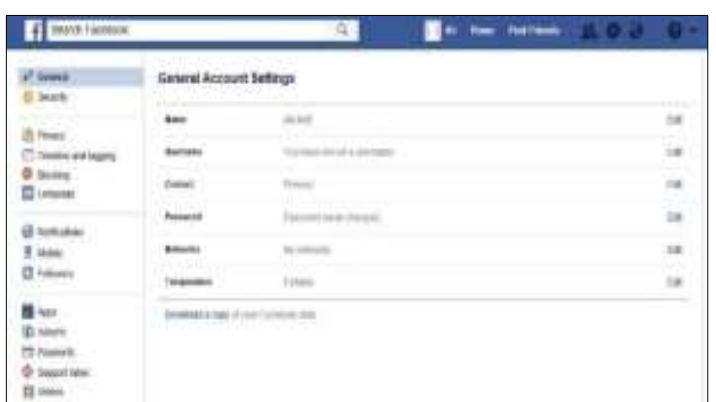

Figure 34. No manage/ deactivation account option provided.

The Twitter website also allows users to create multiple accounts with the same mobile number. When the user logs out from the second account that was created with the same mobile number, it ceases access to both accounts. If the user tries to recover the accounts, it simply displays a message "we found more than one account with that phone number" but no security check or message is displayed during the creation of multiple accounts using the same mobile number.

In the case of using email address instead of mobile number for creation of accounts, it is also observed that both social networking sites provide feasible security with email address login system, the user is unable to create multiple accounts with the same email address in both websites.

\section{Conclusion}

A key security flaw in the login system of both Facebook and Twitter was analysed and discussed. The flaws were determined through mobile numbers that allowed users to create multiple 
accounts with the single mobile number. However, in Twitter, if one account is logged out or closed, then all other subsequent accounts with the same number are also logged off and are not accessible anymore. Whereas, it was also found that multiple accounts created through the same phone number on Facebook, if password is matched with any account that was created with similar mobile number the account will have been overlapped. These security flaws demands urgent attention as well as needs further investigation. Our future research shall include investigation of other security aspects as well as universal usability, especially from cross-cultural [7] and multilingual [8-9] perspectives.

\section{References}

[1] Internet Live Stats (2018). Internet Users. Available: https://www.internetlivestats.com/internet-users/ (retrieved on $15^{\text {th }}$ July 2018)

[2] Aslam, S. (2018, September 18). Facebook by the Numbers: Stats, Demographics \& Fun Facts. Available: https://www.omnicoreagency.com/facebook-statistics/

[3] Aslam, S., (2018, September 18). Twitter by the Numbers: Stats, Demographics \& Fun Facts. Available: https://www.omnicoreagency.com/twitter-statistics/

[4] Janrain, (2014). White Paper: Privacy and Security Advantages of Social Login. (pp. 1-7). Janrain Inc.

[5] Mills, E. (2009, December 23). Using Facebook and Twitter Safely. Available: https://www.cnet.com/news/usingfacebook-and-twitter-safely/

[6] Statista, (2018, July). Most famous social network sites worldwide as of July 2018, ranked by number of active users (in millions). Available: https://www.statista.com/statistics/272014/global-social-networks-ranked-by-number-ofusers/

[7] Mahdi H. Miraz, Maaruf Ali and Peter Excell, "Design for All: Catering for Culturally Diverse Users", in the International Journal of Computer Science and Information Security (IJCSIS), ISSN: 1947-5500, June 2016, Vol. 14, No. 6, pp 516-524, Pittsburgh, PA, USA. Available: https://www.researchgate.net/publication/305441846_Design_ for_All_Catering_for_Culturally_Diverse_Users.

[8] Mahdi H. Miraz, Peter Excell and Maaruf Ali, "User Interface (UI) Design Issues for the Multilingual Users: A Case Study", in the International Journal of Universal Access in the Information Society, Print ISSN: 1615-5289, E- ISSN: 1615-5297, August 2016, Vol. 15, No. 3, pp 431-444, Published by Springer-Verlag, DOI: 10.1007/s10209-014-03975. Available: https://arxiv.org/ftp/arxiv/papers/1709/1709.02737.pdf

[9] Mahdi H. Miraz, Maaruf Ali and Peter Excell, "Multilingual Website Usability Analysis Based on an International User Survey", in the proceedings of the fifth international conference on Internet Technologies and Applications (ITA 13) held at Creative and Applied Research for the Digital Society (CARDS), Glyndŵr University in Wrexham, North East Wales, UK, 10-13 September 2013, ISBN-10: 0-946881-81-2, ISBN-13: 978-0-946881-81-9, pp. $236-244$. Available: https://arxiv.org/ftp/arxiv/papers/1708/1708.05085.pdf.

(C) 2018 by the author(s). Published by Annals of Emerging Technologies in Computing (AETiC), under the terms and conditions of the Creative Commons Attribution (CC BY) license which can be accessed at http://creativecommons.org/licenses/by/4.0/. 